\title{
La profesionalización de la enfermería en Lleida a partir de las Hijas de la Caridad
}

\section{The professionalization of Lleida nursing from the Daughters of Charity}

\section{A profissionalizaçao da Lleida enfermagem da Filhas da Caridade}

Lorena Lourdes Tejero Vidal ${ }^{*}$; Carmen Torres Penella**

* PhD, MSN, RN. Hospital Santa María de Lleida. Profesora Asociada Facultad de Enfermería Universidad de Lleida.

** PhD, MScN, RN. Profesora Titular de Universidad. Facultad de Enfermería Universidad de Lleida.

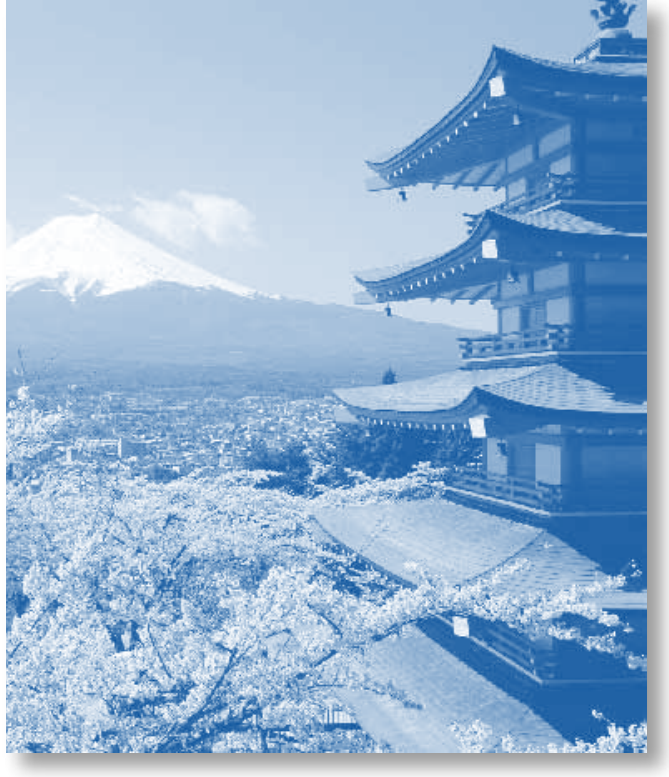

ABSTRACT

The aim of this study is to describe the process of professionalization of nursing in Lleida from the arrival of the Daughters of Charity of St. Vincent de Paul in St. Maria Hospital.

MATERIALS AND METHODS: Historical study by documentary observation technique and analysis of legislative documents. The documents used are: copy of the deed of establishment of the Daughters of Charity of St Maria Hospital (1792) and the Constitutions for the government of the Holy City General Hospital in Lleida (1797).
RESULTS: In the copy of the deed states that the sisters in charge of the regime and care of patients according to the rules of the institute and that both the government and direction inside and outside of them depends on the congregation. In the Constitutions of 1797, credits that have been beneficial to the organization of the hospital to care. Emphasize potential negative disclosures about them.

CONCLUSIONS AND DISCUSSION: The establishment of the Daughters of Charity health care improved patients and health center. The monetary compensation helps us so we can talk of professionalization of nursing.

Keywords: Nursing, Daughters of Charity, Professionalization

\section{RESUMO}

O objetivo deste estudo é descrever o processo de profissionalização da enfermagem em Lleida a partir da chegada das Filhas da Caridade de São Vicente de Paulo no Hospital de Santa Maria.

MATERIAIS E MÉTODOS: Estudo histórico pela técnica de documentário de observação e análise de documentos legislativos. Os documentos utilizados são: cópia da escritura de constituição da Filhas da Caridade do Hos- 
pital de Santa Maria (1792) e as Constituições para o governo da Cidade Santa General Hospital, em Lleida (1797).

RESULTADOS: Na cópia do ato que declara as irmãs responsáveis do regime e dos cuidados dos pacientes de acordo com as regras do instituto e do governo e direção tanto dentro quanto fora deles depende da congregação. Nas Constituições de 1797, os créditos que tenham sido benéficas para a organização do hospital para atendimento. Enfatizar as potenciais divulgações negativas sobre eles.

CONCLUSOES E DISCUSSAO: O estabelecimento das Filhas da Caridade de saúde melhorou e pacientes do centro de saúde. A compensação monetária nos ajuda para que possamos falar de profissionalização da enfermagem.

Palavras-chave: Enfermagem, Filhas da Caridade, Profissionalização.

\section{RESUMEN}

El objetivo del estudio es describir el proceso de profesionalización de la enfermería en Lleida a partir de la llegada de las Hijas de la Caridad de San Vicente de Paúl en el Hospital de Santa María.

MATERIAL Y MÉTODO: Estudio histórico mediante técnica de observación documental y análisis de documentos legislativos. Los documentos utilizados son: copia de la escritura del establecimiento de las Hijas de la Caridad en el Hospital de Santa María (1792) y las Constituciones para el gobierno del Santo Hospital General de la Ciudad de Lérida (1797).

RESULTADOS: En la copia de la escritura se establece que las hermanas se encargarán del régimen y cuidados de los enfermos según las reglas de su instituto; y que tanto el gobierno y dirección interior y exterior de ellas dependerá de la Congregación. En las Constituciones del 1797 se acredita que han resultado beneficiosas tanto para la organización del hospital como para la atención sanitaria. Ponen énfasis en posibles divulgaciones negativas con respecto a ellas.

CONCLUSIONES Y DISCUSIÓN: El establecimiento de las Hijas de la Caridad mejoró la atención sanitaria de los enfermos y la salubridad del centro. La contraprestación económica nos ayuda para que podamos hablar de profesionalización de enfermería.

Palabras clave: Enfermería, Hijas de la Caridad, Profesionalización.

\section{INTRODUCCIÓN}

El Hospital General de Santa María nace de la decisión el 30 de julio de 1453, de situar en intramuros un nuevo hospital que englobase los diversos hospitales existentes hasta el momento en la ciudad de Lleida (Conejo, 2002). No existe unanimidad a la hora de establecer el número de hospitales que fueron fusionados en aquel momento. Según el historiador Pedro Sanahuja (Sanahuja, 1944), serían 7 los fusionados: el de Pere Moliner, Santa Trinitat, Santa Magdalena, Cofradía de Clergues pobres, Sant Tomàs, el de Fillach y el de Vilanova.

El nuevo hospital estaba destinado a la acogida y atención de los pobres de "Cristo" tanto sanos como enfermos, y su financiación se llevaba a cabo con dinero público, donativos particulares entre otros, a través la labor caritativa de la Pía Almoina.

Desde la puesta en marcha de este nuevo hospital, siglo XV hasta finales del siglo XVIII, la práctica de los cuidados de enfermos estuvo en manos de congregaciones eclesiásticas hospitalarias, de los hospitaleros y de sirvientes (Facultat d'Infermería UdL, 2011). Dentro 
de este periodo, aún sin determinar exactamente, el término que designaba el oficio de hospitalero, cambia por el de enfermero/a. Un ejemplo lo tenemos en los libros de cuentas del Hospital del año 1772, en los que se constata el pago del salario a los enfermeros y asistentes; especificando en estos últimos que eran los encargados de realizar las sangrías, la limpieza de las cuadras y la realización de las primeras curas como se ha encontrado en el libro de cuentas del Hospital perteneciente al Fondo de la Pia Almonia.

A mitad del siglo XVIII, la calidad de la práctica de cuidados a la que se sometían los pobres que llegaban al hospital fue decayendo hasta tal punto que las condiciones de insalubridad del mismo fueron tales que nadie quería ingresar en el hospital y las quejas de la vecindad fueron en aumento. La gente miraba el hospital con repugnancia sobre todo por la falta de enfermeros y sirvientes que se dedicasen con esmero a la atención de los enfermos, situación que se ve reflejada en la copia de la escritura del convenio de las Hijas de San Vicente de Paul con el Hospital Santa María de Lleida. Este preocupante hecho llevó a los administradores del hospital a plantearse la forma de subsanar esta situación. Conocedores de la obra de Vicente de Paúl y de sus Hijas de la Caridad dedicadas al cuidado de enfermos y desvalidos, como solución a sus males, propusieron al obispo de Lleida instalar en el hospital las Hijas de la Caridad.

El establecimiento de las Hijas de la Caridad en España data de 1790 después de un proceso de aprendizaje de seis postulantes españolas que marcharon en 1782 a Narbona para abrazar la Congregación fundada por San Vicente de Paül y aspirar a ser Hijas de la Caridad (Hernandez, 1988).

Con la revolución francesa, estas seis Hijas de la Caridad españolas regresan a España y se instalan en un primer momento en Barcelona para asistir a los enfermos del Hospital de Santa Creu. Por razones aún por dilucidar, no todas las hermanas se quedaron en Barcelona; cuatro de ellas, se instalaron en Lleida para hacerse cargo de la atención sanitaria de los enfermos en el antiguo Hospital de Santa María de Lleida en 1792, debido a las buenas referencias que se tenía en aquel momento de la atención que suministraban las Hijas de la Caridad a los enfermos.

El Permiso Real para el establecimiento de las mismas en el Hospital Santa María data el 11 de Agosto de 1792. La escritura del contrato para su establecimiento se autoriza el $30 \mathrm{de}$ noviembre de 1792 y el día 3 de Diciembre del mismo año empiezan su servicio en el hospital. Servicio que se desarrolla a lo largo de dos siglos hasta la actualidad.

\section{OBJETIVOS}

El objetivo del estudio es el de analizar el inicio del proceso de profesionalización de la enfermería en Lleida comprendido desde la llegada de las Hijas de la Caridad de San Vicente de Paúl en 1792 en el Hospital de Santa María, hasta las Constituciones del 1797.

\section{MATERIAL Y MÉTODO}

Se trata de un estudio histórico, a partir de fuentes documentales y bibliográficas. Las técnicas utilizadas son la observación documental y el análisis de texto de documentos legales administrativos referentes al gobierno del hospital y a la vinculación de las Hijas de Caridad en el mismo.

Los documentos utilizados para el estudio son dos: la copia de la escritura del establecimiento de las Hijas de la Caridad en el Hospital de Santa María en 1792 y las Constituciones 
para el gobierno del Santo Hospital General de la Ciudad de Lérida del 1797 del Archivo del Institut d' Estudis Ilerderdencs y del Archivo Municipal de Lleida

Elementos de estudio: Las condiciones laborales y las competencias profesionales en cuidados de enfermería de las Hermanas de la Caridad en el transcurso de estos primeros cinco años de estancia en el Hospital, así como otro personal de enfermería en las figuras de practicante y enfermero.

\section{RESULTADOS}

La copia de la escritura del establecimiento de las Hijas de la Caridad en el Hospital de Santa María con fecha 30 de noviembre de 1792, empieza destacando la situación precaria por la que pasaba el Hospital en aquellos años, y cuáles fueron los motivos para la solicitud de implantación de la congregación en el mismo. Se trata del primer contrato que existe en el estado español del las Hijas de la Caridad con una institución hospitalaria. Establece que las hermanas de la caridad se encargarán del régimen y cuidados de los pobres enfermos según las reglas de su Instituto y delimita que el gobierno exterior del hospital dependerá de los señores administradores. En el cuarto punto de la contrata establecen que "tanto el gobierno $y$ dirección interior y exterior de ellas solo deben estar inmediatamente sujetas a la Congregación de la Misión, es decir al Visitador de la misma Congregación de la provincia de España, y del Superior de la Casa de la Congregación de la Misión de Lérida”. Fue este punto el más polémico y que dejaron claro, ya que había creado conflictos a su paso por Barcelona.

En el momento de la contrata, el número de hermanas de la caridad que se autorizan es el de cuatro, pero se deja abierta la posibilidad de aumentar su número en función de las ne- cesidades del hospital. La provisión por parte de los administradores del hospital de víveres, vestiduras propias de la congregación y la asistencia sanitaria a las hermanas, se determina como uno de los requisitos dentro de la contrata.

Las primeras constituciones conocidas del Hospital de Santa María datan del 1797. Se estructuran en 7 apartados, siendo la constitución primera la delimitación del régimen, composición y organización de la junta para el gobierno del Hospital.

Es en la constitución III se deja constancia de los beneficios que la instalación de las hermanas de la caridad en el hospital aporta. De ellas dicen: "la experiencia ha acreditado su utilidad, y las ventajas que logra el Hospital, ya en la mejor asistencia de los Pobres, ya en el aseo y limpieza de las camas y habitaciones, ya en el cuidado de los niños expósitos, ya en el manejo económico y fiel de lo que se distribuye por sus manos". Son además las encargadas de la limpieza de las "quadras" y de velar por las noches a los enfermos.

En la constitución V, se delimita la figura del practicante como un asistente de los médicos y de los cirujanos. "Debe ser un mozo hábil $e$ inteligente". Será él el encargado hasta ese momento de distribuir las medicinas recetadas, siempre con la cautela de la observación de los enfermos.

En la constitución VIII, se presenta la figura del enfermero. Destacando al inicio de esta apartado lo siguiente "Aunque las Hermanas de la Caridad son propiamente las Enfermeras que asisten a los Enfermos, repartiéndoles la comi$d a$, haciendo las camas, y cuidando del aseo y limpieza de las Quadras, y velar de noche, alternando entre ellas mismas según ordena la Presidente; hay ciertos oficios que no pueden hacer por sí, ni es decente que los hagan con los de otro 
sexo. Por lo mismo la Junta debe nombrar un Enfermero, que estará baxo las órdenes de las Hermanas...”. El enfermero, según las constituciones, deberá ser un "hombre de buenas costumbres, y sin aquellos vicios groseros que son bastantes comunes..."

\section{CONCLUSIONES Y DISCUSIÓN}

En el transcurso de un lustro, el antiguo hospital de Santa María experimento mejoras en la organización y atención sanitaria de los enfermos que eran atendidos en el mismo. La pregunta es, ¿sson las Hermanas de la Caridad responsables de dichas mejoras? Desde nuestro punto de vista, la aportación que hicieron debido a su bagaje de conocimientos, habilidades y actitudes con las que desarrollaban su gestión y atención de cuidados directos a los enfermos así lo ratifica. En ambos documentos, pueden verse como los administradores eran conscientes de la repercusión que supondrían las aportaciones de dicha congregación al hospital. Con el paso del tiempo, pudieron objetivar los resultados obtenidos.

El hecho de que la actividad que desarrollaban perdurase en el tiempo (concepto de durabilidad) y que hubiese una contraprestación económica, hace que se pueda hablar de profesión enfermera, como establece el profesor Hall. Estos dos factores unidos a la constitución de un servicio a la sociedad, la disposición de un cuerpo de conocimientos, el establecimiento de normas propias y la posibilidad de gobernarse a uno mismo (entre otros), son requisitos necesarios para poder hablar del proceso de profesionalización (Hernandez, 2006).

Hay que tener en cuenta que las hermanas no estaban solas para llevar a cabo el arduo trabajo de cuidar a enfermos, sino que disponían tanto de practicantes como de enfermeros; figuras dentro del hospital, que con anterioridad a la llegada de las hermanas habían sido los únicos que habían realizado los cuidados a los enfermos. Este hecho, pudo ser motivo de recelo por la pérdida de poder que suponer, como se ve reflejado en las Constituciones del Hospital del 1797.

\section{BIBLIOGRAFÍA}

- Archivo Institut d'Estudis Ilerdencs. Madriguera, D.I. (notarios). Copia de escritura de convenio de las Hijas de San Vicente de Paul con el Hospital de Santa María de Lérida. Lérida 30 noviembre de 1792. Legajo núm. 47. SAIEI.

- Archivo Institut d'Estudis Ilerdencs. Constituciones para el Gobierno del Santo Hospital General de la Ciudad de Lerida, hechas de orden del supremo consejo de Castilla con arreglo a la nueva planta y forma aprobada por este supremo tribunal. Madrid. Oficina de Don Blas Román. 1797.

- Archivo Municipal de Lleida. Fons Pia Almonia, Vol 097/337. Llibre de comptes de l'Hospital General de Lleida.

- Conejo da Pena, A. (2002) L'Antic Hospital de Santa María. Seu de L'Institut d'Estudis Ilerdencs. Ed. Institut d'Estudis Ilerdencs

- Facultat d'Infermeria de la Universitat de Lleida. (2011) 25 anys treballant amb cura. Apunts històrics de l'Escola d'Infermeria de Lleida. Ed. Universitat de Lleida; 53-72

- Hall, C.M. ¿Quién controla la profesión? Rol de la Asociación Profesional. XV Congreso Cuadrienal del CIE. México, 1973.

- Hernandez Martin, F (2006). Las Hijas de la Caridad en la profesionalización de Enfermería. Rev. Cultura de los Cuidados; 10 (20): 39-49.

- Hernandez Zapatel, C. (1988) Las hijas de la Caridad en España 1782-1856. Ed. CEME; 93-121.

- Sanhuja, P. (1944) Historia de la beneficencia en Lérida: La Almonia de la Catedral. Vol I. Lleida: Institut d'Estudis Ilerdencs. 